\title{
Impact of Wind Guess on the Tracking of Atmospheric Motion Vectors
}

\author{
RÉGIS BORDE \\ EUMETSAT, Darmstadt, Germany \\ JAVIER GARCÍA-PEREDA \\ AEMET, Madrid, Spain
}

(Manuscript received 23 April 2013, in final form 6 November 2013)

\begin{abstract}
The goal of this paper is to show the impact of the use of the wind guess (WG) in atmospheric motion vector (AMV) extraction schemes. The study has been performed using the Satellite Application Facility on Support to Nowcasting and Very Short Range Forecasting (NWCSAF) High Resolution Winds AMV software. Target box sizes varying from $8 \times 8$ to $40 \times 40$ pixels and temporal gaps varying from 5 to 60 min have been considered for two configurations that use WG and do not use the wind guess (NWG) to locate the search area in the tracking process. AMVs have been extracted for four different Meteosat Second Generation (MSG) Spinning Enhanced Visible and Infrared Imager (SEVIRI) channels [high-resolution visible (HRVIS), visible $0.8 \mu \mathrm{m}$ (VIS0.8), water vapor $6.2 \mu \mathrm{m}$ (WV6.2), and infrared $10.8 \mu \mathrm{m}$ (IR10.8)] over the European and Mediterranean area for a 6-month period (January-June 2010). The AMVs' performances have been tested against radiosonde wind observations and ECMWF NWP model wind analysis.

The results show an impact on the amount of valid AMVs extracted by each configuration. Not using the wind guess produces more valid AMVs when large target boxes and short temporal gaps are used. It is the opposite when small target boxes and long temporal gaps are used. The results also show a general increase in the mean AMV speed, and a general reduction of the normalized bias and the normalized root-mean-square vector difference for all the tested channels and configurations, when the wind guess is not used to locate the search area.
\end{abstract}

\section{Introduction}

Atmospheric motion vectors (AMVs) are derived from tracking clouds or water vapor features in consecutive satellite images. They are the only upper-wind observations with good global coverage for the tropics and midlatitudes, especially over the large ocean areas, and they constitute a significant part of the observation data assimilated in numerical weather prediction (NWP) models. When using the cross-correlation process, the first step of the AMV extraction scheme selects a target to be tracked at a selected grid point. The motion is derived by matching the position that best corresponds to this target in the later image. However, it is a common process to use wind guess (WG) information to locate the search area in the later image before the matching (Velden et al.

Corresponding author address: Régis Borde, EUMETSAT, Eumetsat Allee 1, D-64295 Darmstadt, Germany.

E-mail: regis.borde@eumetsat.int
1997; Bedka and Mecikalski 2005; Bresky et al. 2012). This allows the use of smaller search areas, which speeds up the process and reduces the computing time necessary to derive operationally the AMVs. This strategy requires estimating the altitude of the AMV before the matching process, to search in the nearest NWP forecast profiles for the speed and direction that correspond to the estimated altitude, and to use this information to locate the search area in the later image.

The AMV height assignment step is an important source of error in the AMV extraction scheme due to the sensitivity of the methods used [water vapor (WV) intercept method, Schmetz et al. (1993); $\mathrm{CO}_{2}$ slicing method, Menzel et al. (1983)] to local atmospheric parameters like cloud optical thickness, cloud microphysics, cloud depth, and multilayer cloud situations (Borde and Dubuisson 2010). Setting an incorrect AMV altitude before the matching process can introduce an error that is propagated to the tracking step, locating the search box in an area where the real matching solution 


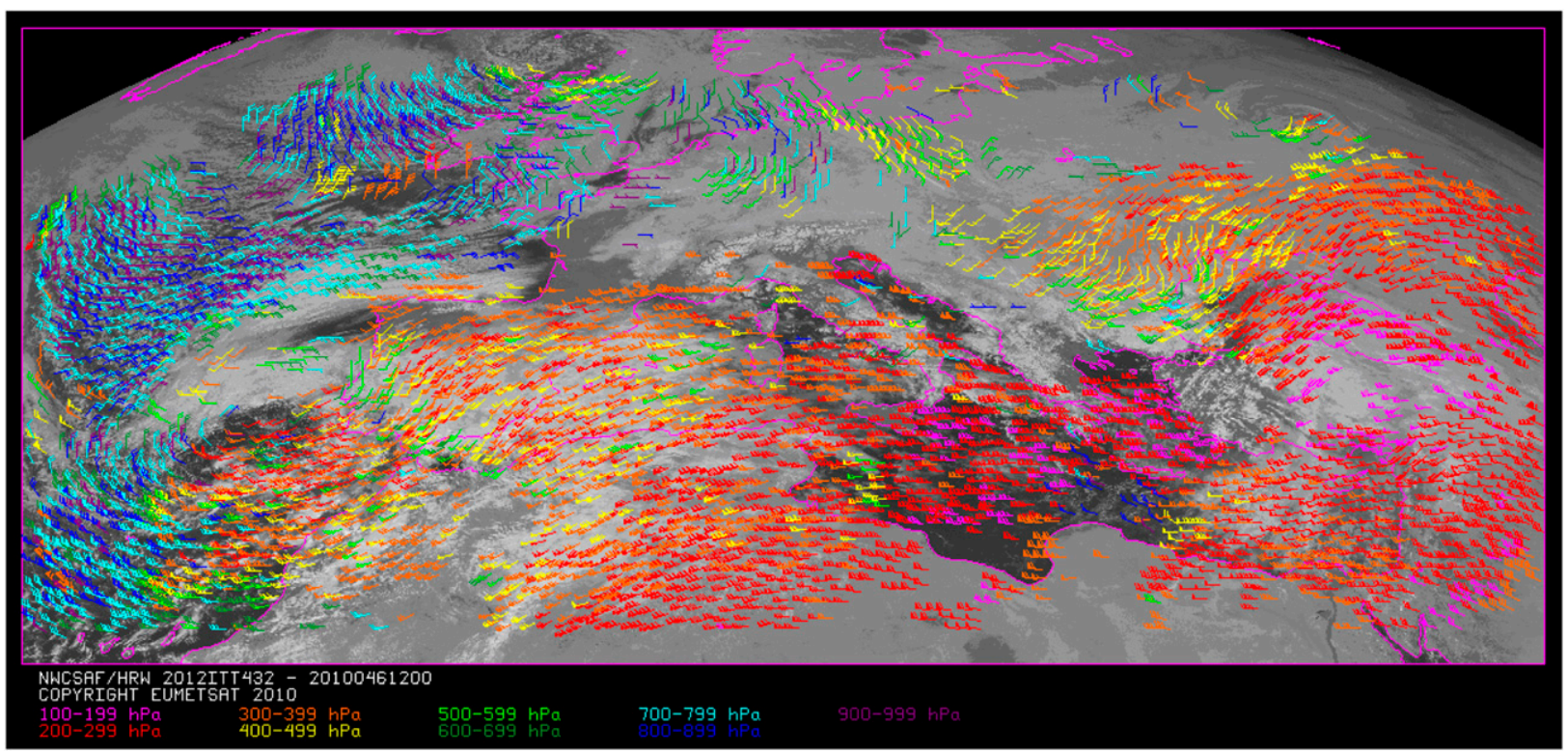

FIG. 1. Display of AMVs over the studied area at 1200 UTC 15 Feb 2010. AMVs have been extracted not using wind guess to locate the search area with a $24 \times 24$ pixel target box and a 15-min temporal gap between images.

cannot be found. Sometimes the whole process can also result in simply replicating the model field, artificially creating an apparently good AMV in areas where the matching is really uncertain.

This paper shows the impact of the use of the wind guess in the tracking step of AMV extraction. During this study, the High Resolution Winds AMV extraction software (HRW; SAFNWC/MSG HRW) by the Satellite Application Facility on Support to Nowcasting and Very Short Range Forecasting (NWCSAF) has been used in two parallel configurations, using and not using the wind guess to locate the search area. The first part of this paper describes the NWC SAF/HRW software and the conditions applied during this study. The second part shows the wind guess impact on the AMV software performance and discusses the results.

\section{General description of the data}

The SAFNWC/MSG HRW software used during this study provides high-density sets of AMVs from Meteosat Second Generation (MSG) Spinning Enhanced Visible and Infrared Imager (SEVIRI) images. The HRW version 3.2 (v3.2) released to users in February 2012 allows for the extraction of AMVs using seven different MSG/ SEVIRI channels: high-resolution visible (HRVIS), visible $0.6 \mu \mathrm{m}$ (VIS0.6), visible $0.8 \mu \mathrm{m}$ (VIS0.8), infrared $10.8 \mu \mathrm{m}$ (IR10.8), infrared $12.0 \mu \mathrm{m}$ (IR12.0), water vapor $6.2 \mu \mathrm{m}$ (WV6.2), and water vapor 7.3 $\mu \mathrm{m}$ (WV7.3; GarcíaPereda 2013a,b).
The cross-correlation contribution (CCC) method (Borde and Oyama 2008; Borde et al. 2014) is used to select the pixels used for the calculation of the AMV altitude. The European Organisation for the Exploitation of Meteorological Satellites (EUMETSAT) quality control process (Holmlund 1998) is used to define the quality of the AMVs. A quality index (QI) from 0 to 100 is assigned to each AMV that considers the spatial consistency with neighboring winds extracted in the same image, the temporal consistency with winds in the previous image, and the consistency with the NWP fields. Only AMVs that have a QI larger than $85 \%$ are considered in the framework in this study.

Two configurations of the NWC SAF/HRW software have been considered, one using the wind guess to locate the search area in the later image (noted as WG) and the second one not using the wind guess (noted as NWG). AMVs have been extracted from the Meteorological Satellite-8 (Meteosat-8)'s MSG/SEVIRI rapid-scan HRVIS, VIS0.8, IR10.8, and WV6.2 channels, over the European and Mediterranean region $(772 \times 1856$ SEVIRI low-resolution pixels centered in $40.5^{\circ} \mathrm{N}, 11^{\circ} 1 \mathrm{E}$; Fig. 1), for 132 days between January and June 2010 and using the three consecutive images that end at 1200 UTC. AMVs are calculated for the two pairs of images (first one vs second one, second one vs third one), with the last one providing the final AMV product. Twelve different configurations with target boxes varying from $8 \times 8$ to $40 \times 40$ pixels and temporal gaps between 5 and $60 \mathrm{~min}$ have been considered. 
TABLE 1. Number of AMVs for all configurations and MSG/SEVIRI channels with different target boxes between $8 \times 8$ and $40 \times 40$ pixels, and temporal gaps between 5 and $60 \mathrm{~min}$, for WG (left) and NWG (middle). Amount of AMVs for the NWG configuration with respect to the WG configuration for the different experiments is also shown (rightmost column).

\begin{tabular}{|c|c|c|c|c|c|c|c|c|c|}
\hline & $8 \times 8$ & $24 \times 24$ & $40 \times 40$ & $8 \times 8$ & $24 \times 24$ & $40 \times 40$ & $8 \times 8$ & $24 \times 24$ & $40 \times 40$ \\
\hline & \multicolumn{3}{|c|}{ HRVIS WG } & \multicolumn{3}{|c|}{ HRVIS NWG } & \multicolumn{3}{|c|}{ HRVIS \%Diff } \\
\hline 5 & 3893066 & 455067 & 119616 & 2378578 & 449922 & 121916 & -39 & -1 & +2 \\
\hline 15 & 1461508 & 270289 & 77556 & 409803 & 226734 & 74716 & -72 & -16 & -4 \\
\hline 30 & 496713 & 86072 & 22787 & 85698 & 52267 & 19457 & -83 & -39 & -15 \\
\hline \multirow[t]{2}{*}{60} & 258816 & 16064 & 3850 & 29737 & 4841 & 2030 & -89 & -70 & -47 \\
\hline & \multicolumn{3}{|c|}{ VIS08 WG } & \multicolumn{3}{|c|}{ VIS08 NWG } & \multicolumn{3}{|c|}{ VIS08 \% Diff } \\
\hline 5 & 447656 & 81596 & 25022 & 874966 & 114314 & 30410 & +95 & +40 & +22 \\
\hline 15 & 1177944 & 185903 & 51260 & 673459 & 181237 & 51287 & -43 & -3 & +0 \\
\hline 30 & 501367 & 88858 & 25417 & 124041 & 75284 & 23552 & -75 & -15 & -7 \\
\hline \multirow[t]{2}{*}{60} & 137883 & 25181 & 6644 & 16933 & 13014 & 4507 & -88 & -48 & -32 \\
\hline & \multicolumn{3}{|c|}{ IR108 WG } & \multicolumn{3}{|c|}{ IR108 NWG } & \multicolumn{3}{|c|}{ IR108 \%Diff } \\
\hline 5 & 431851 & 96001 & 27536 & 792454 & 133616 & 36241 & +84 & +39 & +32 \\
\hline 15 & 1127246 & 214725 & 60104 & 741832 & 226417 & 66245 & -34 & +5 & +10 \\
\hline 30 & 550325 & 126924 & 37,602 & 167363 & 117118 & 37526 & -70 & -8 & -0 \\
\hline \multirow[t]{2}{*}{60} & 147646 & 31449 & 8316 & 21157 & 16739 & 5521 & -86 & -47 & -34 \\
\hline & \multicolumn{3}{|c|}{ WV062 cloudy WG } & \multicolumn{3}{|c|}{ WV062 cloudy NWG } & \multicolumn{3}{|c|}{ WV062 cloudy \%Diff } \\
\hline 5 & 344038 & 140886 & 47119 & 518039 & 159042 & 49869 & +51 & +13 & +6 \\
\hline 15 & 658168 & 191638 & 59515 & 445357 & 197707 & 63230 & -32 & +3 & +6 \\
\hline 30 & 362948 & 124127 & 41652 & 137374 & 116386 & 43283 & -62 & -6 & +4 \\
\hline \multirow[t]{2}{*}{60} & 118515 & 43731 & 16338 & 26579 & 25331 & 12432 & -78 & -42 & -24 \\
\hline & \multicolumn{3}{|c|}{ WV062 clear-air WG } & \multicolumn{3}{|c|}{ WV062 clear-air NWG } & \multicolumn{3}{|c|}{ WV062 clear-air \% Diff } \\
\hline 5 & 67706 & 45083 & 17944 & 60606 & 46776 & 19310 & -10 & +4 & +8 \\
\hline 15 & 106687 & 69374 & 28279 & 56129 & 68126 & 28899 & -47 & -2 & +2 \\
\hline 30 & 87138 & 66974 & 30041 & 33729 & 56743 & 28646 & -61 & -15 & -5 \\
\hline 60 & 75168 & 53468 & 26278 & 21651 & 30616 & 18660 & -71 & -43 & -29 \\
\hline
\end{tabular}

The SAFNWC/MSG cloud products (cloud mask, cloud type, and cloud-top temperature and height) and the SAFNWC/MSG HRW product were obtained considering the 0000 UTC European Centre for MediumRange Weather Forecasts (ECMWF) NWP model forecast in the calculations. The model data used contain 12 vertical levels $(1000,925,850,700,500,400,300,250$, $200,150,100$, and $70 \mathrm{hPa}$ ) of geopotential, wind components, and air and surface temperature with a $0.125^{\circ}$ resolution. The final AMVs have been compared against 1200 radiosonde (RS) wind observations and the 1200 UTC ECMWF NWP model wind analysis (MA). The comparison of AMVs against collocated radiosonde observations follows the Coordination Group for Meteorological Satellites (CGMS) criteria defined at the third International Wind Workshop (1996) in Ascona, Switzerland, for the validation of satellite winds. These are the standard criteria used by AMV producers to monitor the quality of the AMV product (with a horizontal distance of $\mathrm{AMV} / \mathrm{RS}<150 \mathrm{~km}$ and a vertical distance of $\mathrm{AMV} / \mathrm{RS}<25 \mathrm{hPa}$ ). For these reasons, only a small proportion of the total number of AMVs is successfully compared to radiosonde winds.

All parameters and methods considered in the WG and NWG cases were similar except the search area size, which needs to be larger in NWG cases to permit the search for fast tracers in any direction around its location in the initial image. The configuration used guarantees that wind speeds of at most $300 \mathrm{~km} \mathrm{~h}^{-1}\left(83 \mathrm{~m} \mathrm{~s}^{-1}\right)$ per component can be detected in the NWG configuration, while wind speed differences with respect to the NWP wind guess of at most $108 \mathrm{~km} \mathrm{~h}^{-1}\left(30 \mathrm{~m} \mathrm{~s}^{-1}\right)$ per component can be detected in the WG cases. This value has been chosen to reduce the computing time of the HRWv3.2 algorithm below 2 min for the "European and Mediterranean Sea region," considering a specific configuration with a 15-min temporal gap and a $24 \times 24$ pixel tracer size in the operational environment. For example, for a temporal gap between images of $15 \mathrm{~min}$ and a target size of $24 \times 24$ pixels, the size of the search area corresponds to $174 \times 174$ pixels in the HRVIS and $74 \times 74$ pixels in the low-resolution images (VIS0.8, IR10.8, and WV6.2) in the NWG cases. It corresponds to $78 \times 78$ pixels in the high-resolution images and $42 \times 42$ pixels in the low-resolution images in the WG cases. The computational cost of the WG configuration was around 7 days of continuous running; the computational cost of the NWG configuration was around 18 days of continuous running - therefore, about $170 \%$ longer. 
TABLE 2. As in Table 1, but for the mean AMV tracking correlation (\%). Difference (Diff) in the mean AMV tracking correlation, for the NWG configuration respect to the WG configuration for the different experiments is also shown (rightmost column).

\begin{tabular}{|c|c|c|c|c|c|c|c|c|c|}
\hline & $8 \times 8$ & $24 \times 24$ & $40 \times 40$ & $8 \times 8$ & $24 \times 24$ & $40 \times 40$ & $8 \times 8$ & $24 \times 24$ & $40 \times 40$ \\
\hline & \multicolumn{3}{|c|}{ HRVIS WG } & \multicolumn{3}{|c|}{ HRVIS NWG } & \multicolumn{3}{|c|}{ HRVIS Diff } \\
\hline 5 & $91.6 \%$ & $92.5 \%$ & $93.2 \%$ & $92.7 \%$ & $92.6 \%$ & $93.2 \%$ & $+1.1 \%$ & $+0.1 \%$ & $+0.0 \%$ \\
\hline 15 & $91.0 \%$ & $88.8 \%$ & $88.8 \%$ & $92.4 \%$ & $89.1 \%$ & $88.9 \%$ & $+1.4 \%$ & $+0.3 \%$ & $+0.1 \%$ \\
\hline 30 & $90.7 \%$ & $87.2 \%$ & $87.0 \%$ & $92.2 \%$ & $87.8 \%$ & $87.1 \%$ & $+1.5 \%$ & $+0.6 \%$ & $+0.1 \%$ \\
\hline 60 & $90.7 \%$ & $\begin{array}{c}87.0 \% \\
\text { VIS08 W }\end{array}$ & $86.2 \%$ & \multicolumn{3}{|c|}{ VIS08 NWG } & $+1.6 \%$ & $\begin{array}{c}+0.7 \% \\
\text { VIS08 Dif }\end{array}$ & $+0.0 \%$ \\
\hline 5 & $93.1 \%$ & $94.6 \%$ & $95.1 \%$ & $92.6 \%$ & $94.1 \%$ & $94.9 \%$ & $-0.5 \%$ & $-0.5 \%$ & $-0.2 \%$ \\
\hline 15 & $90.0 \%$ & $89.8 \%$ & $90.3 \%$ & $90.8 \%$ & $89.8 \%$ & $90.3 \%$ & $+0.8 \%$ & $-0.0 \%$ & $-0.0 \%$ \\
\hline 30 & $89.2 \%$ & $87.7 \%$ & $87.8 \%$ & $90.6 \%$ & $87.8 \%$ & $87.8 \%$ & $+1.4 \%$ & $+0.1 \%$ & $+0.0 \%$ \\
\hline 60 & \multicolumn{3}{|c|}{ IR108 WG } & \multicolumn{3}{|c|}{ IR108 NWG } & \multicolumn{3}{|c|}{ IR108 Diff } \\
\hline 5 & $94.8 \%$ & $96.0 \%$ & $96.5 \%$ & $94.4 \%$ & $95.7 \%$ & $96.4 \%$ & $-0.4 \%$ & $-0.3 \%$ & $-0.1 \%$ \\
\hline 15 & $91.7 \%$ & $91.5 \%$ & $91.9 \%$ & $92.5 \%$ & $91.5 \%$ & $91.9 \%$ & $+0.8 \%$ & $-0.0 \%$ & $-0.0 \%$ \\
\hline 30 & $90.4 \%$ & $88.2 \%$ & $87.9 \%$ & $91.8 \%$ & $88.2 \%$ & $87.9 \%$ & $+1.4 \%$ & $+0.0 \%$ & $+0.0 \%$ \\
\hline \multirow[t]{2}{*}{60} & $90.3 \%$ & $86.9 \%$ & $86.5 \%$ & $91.9 \%$ & $87.2 \%$ & $86.5 \%$ & $+1.6 \%$ & $+0.3 \%$ & $+0.0 \%$ \\
\hline & \multicolumn{3}{|c|}{ WV062 cloudy WG } & \multicolumn{3}{|c|}{ WV062 cloudy NWG } & \multicolumn{3}{|c|}{ WV062 cloudy Diff } \\
\hline 5 & $92.9 \%$ & $94.3 \%$ & $95.6 \%$ & $92.5 \%$ & $94.1 \%$ & $95.6 \%$ & $-0.4 \%$ & $-0.2 \%$ & $+0.0 \%$ \\
\hline 15 & $90.6 \%$ & $91.3 \%$ & $92.5 \%$ & $91.2 \%$ & $91.4 \%$ & $92.5 \%$ & $+0.6 \%$ & $+0.1 \%$ & $-0.0 \%$ \\
\hline 30 & $89.5 \%$ & $88.9 \%$ & $89.6 \%$ & $90.5 \%$ & $89.0 \%$ & $89.5 \%$ & $+1.0 \%$ & $+0.1 \%$ & $-0.1 \%$ \\
\hline \multirow[t]{2}{*}{60} & $89.3 \%$ & $88.4 \%$ & $89.0 \%$ & $90.7 \%$ & $88.8 \%$ & $89.0 \%$ & $+1.4 \%$ & $+0.4 \%$ & $-0.0 \%$ \\
\hline & \multicolumn{3}{|c|}{ WV062 clear-air WG } & \multicolumn{3}{|c|}{ WV062 clear-air NWG } & \multicolumn{3}{|c|}{ WV062 clear-air Diff } \\
\hline 5 & $91.0 \%$ & $92.4 \%$ & $94.6 \%$ & $90.8 \%$ & $92.4 \%$ & $94.6 \%$ & $-0.2 \%$ & $+0.0 \%$ & $-0.0 \%$ \\
\hline 15 & $90.3 \%$ & $91.5 \%$ & $93.5 \%$ & $91.2 \%$ & $91.6 \%$ & $93.4 \%$ & $+0.9 \%$ & $+0.1 \%$ & $-0.1 \%$ \\
\hline 30 & $90.4 \%$ & $90.9 \%$ & $92.4 \%$ & $91.9 \%$ & $91.2 \%$ & $92.5 \%$ & $+1.5 \%$ & $+0.3 \%$ & $+0.1 \%$ \\
\hline 60 & $90.9 \%$ & $90.8 \%$ & $91.8 \%$ & $92.8 \%$ & $91.5 \%$ & $92.0 \%$ & $+1.9 \%$ & $+0.7 \%$ & $+0.2 \%$ \\
\hline
\end{tabular}

The WG configuration must also estimate the altitude of the cloud feature present in the target box before the tracking, so that the ECMWF NWP forecast wind speed and direction interpolated to this altitude are used to locate the search area center in the later image. The cloud feature altitude estimation is based on the interpolation of the NWP model temperature to the temperature estimated for the cloud base $\left(T_{\text {base }}=T_{\text {mean }}+k \sigma_{T}\right.$,

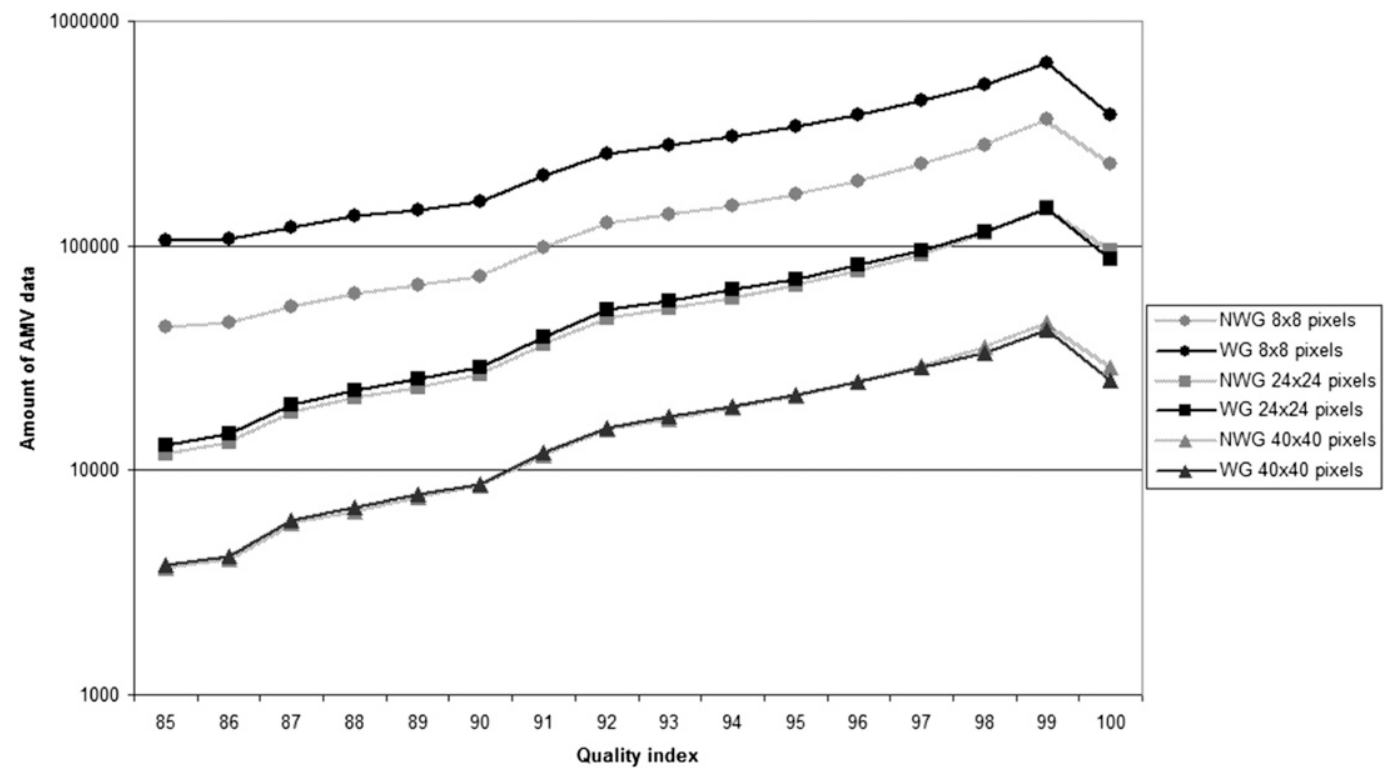

FIG. 2. Distributions of the number of AMVs extracted for all the WG and NWG configurations that use 15-min temporal gaps and $8 \times 8,24 \times 24$, and $40 \times 40$ pixel target boxes, respectively. The $Y$ axis is in logarithm scale. 
TABLE 3. As in Table 1, but for the mean AMV speed $\left(\mathrm{m} \mathrm{s}^{-1}\right)$. Percent difference in the mean AMV speed for the NWG configuration with respect to the WG configuration for the different experiments is also shown (rightmost column).

\begin{tabular}{|c|c|c|c|c|c|c|c|c|c|}
\hline & $8 \times 8$ & $24 \times 24$ & $40 \times 40$ & $8 \times 8$ & $24 \times 24$ & $40 \times 40$ & $8 \times 8$ & $24 \times 24$ & $40 \times 40$ \\
\hline & \multicolumn{3}{|c|}{ HRVIS WG } & \multicolumn{3}{|c|}{ HRVIS NWG } & \multicolumn{3}{|c|}{ HRVIS \%Diff } \\
\hline 5 & 19.0 & 18.1 & 18.8 & 19.2 & 18.4 & 19.1 & $+1 \%$ & $+2 \%$ & $+1 \%$ \\
\hline 15 & 19.5 & 16.6 & 16.4 & 22.1 & 17.7 & 17.5 & $+13 \%$ & $+7 \%$ & $+7 \%$ \\
\hline 30 & 23.4 & 15.3 & 14.0 & 31.7 & 17.0 & 16.0 & $+35 \%$ & $+11 \%$ & $+14 \%$ \\
\hline \multirow[t]{2}{*}{60} & 27.9 & 15.7 & 11.8 & 44.6 & 18.5 & 13.0 & $+60 \%$ & $+18 \%$ & $+10 \%$ \\
\hline & \multicolumn{3}{|c|}{ VIS08 WG } & \multicolumn{3}{|c|}{ VIS08 NWG } & \multicolumn{3}{|c|}{ VIS08 \%Diff } \\
\hline 5 & 23.8 & 22.7 & 22.4 & 23.8 & 22.7 & 22.4 & $+0 \%$ & $-0 \%$ & $+0 \%$ \\
\hline 15 & 16.5 & 14.9 & 14.5 & 17.6 & 15.8 & 15.3 & $+7 \%$ & $+6 \%$ & $+5 \%$ \\
\hline 30 & 16.6 & 13.6 & 12.9 & 19.9 & 14.8 & 13.8 & $+20 \%$ & $+9 \%$ & $+7 \%$ \\
\hline \multirow[t]{2}{*}{60} & 19.7 & 12.3 & 11.8 & 27.8 & 13.7 & 12.6 & $+41 \%$ & $+11 \%$ & $+7 \%$ \\
\hline & \multicolumn{3}{|c|}{ IR108 WG } & \multicolumn{3}{|c|}{ IR108 NWG } & \multicolumn{3}{|c|}{ IR108 \%Diff } \\
\hline 5 & 26.7 & 25.7 & 25.4 & 27.2 & 26.7 & 26.9 & $+2 \%$ & $+4 \%$ & $+6 \%$ \\
\hline 15 & 20.8 & 19.6 & 19.5 & 22.8 & 22.0 & 22.3 & $+10 \%$ & $+12 \%$ & $+15 \%$ \\
\hline 30 & 21.2 & 19.1 & 18.6 & 25.6 & 21.9 & 21.5 & $+21 \%$ & $+15 \%$ & $+16 \%$ \\
\hline \multirow[t]{2}{*}{60} & 24.3 & 19.1 & 17.9 & 33.8 & 22.7 & 20.6 & $+39 \%$ & $+19 \%$ & $+16 \%$ \\
\hline & \multicolumn{3}{|c|}{ WV062 cloudy WG } & \multicolumn{3}{|c|}{ WV062 cloudy NWG } & \multicolumn{3}{|c|}{ WV062 cloudy \%Diff } \\
\hline 5 & 30.6 & 29.1 & 29.1 & 30.3 & 29.1 & 29.1 & $-1 \%$ & $+0 \%$ & $-0 \%$ \\
\hline 15 & 26.1 & 24.9 & 25.2 & 27.8 & 26.7 & 27.2 & $+7 \%$ & $+7 \%$ & $+8 \%$ \\
\hline 30 & 24.3 & 22.6 & 22.6 & 28.7 & 25.6 & 25.7 & $+18 \%$ & $+14 \%$ & $+14 \%$ \\
\hline \multirow[t]{2}{*}{60} & 25.4 & 21.1 & 20.4 & 33.2 & 24.2 & 23.4 & $+31 \%$ & $+15 \%$ & $+15 \%$ \\
\hline & \multicolumn{3}{|c|}{ WV062 clear-air WG } & \multicolumn{3}{|c|}{ WV062 clear-air NWG } & \multicolumn{3}{|c|}{ WV062 clear-air \%Diff } \\
\hline 5 & 30.1 & 25.8 & 24.4 & 30.0 & 25.7 & 24.1 & $-0 \%$ & $-1 \%$ & $-1 \%$ \\
\hline 15 & 25.0 & 21.2 & 20.6 & 27.5 & 22.8 & 22.0 & $+10 \%$ & $+7 \%$ & $+7 \%$ \\
\hline 30 & 23.1 & 19.8 & 19.3 & 27.1 & 21.6 & 21.0 & $+17 \%$ & $+9 \%$ & $+9 \%$ \\
\hline 60 & 22.8 & 18.8 & 18.0 & 26.8 & 19.5 & 18.8 & $+18 \%$ & $+4 \%$ & $+4 \%$ \\
\hline
\end{tabular}

where $T_{\text {mean }}$ is the mean brightness temperature, $k$ is a constant, and $\sigma_{T}$ is the standard deviation of the brightness temperatures) or for the cloud top (brightness temperature of the coldest class in the tracer temperature histogram, represented at least by three pixels, after histogram smoothing), depending on the cloud type related to the tracer (García-Pereda 2013a). The center of the search area in the later image is set to the position where the feature in the initial image is expected to be found, assuming that the estimated NWP wind guess and altitude are correct.

The final AMV altitude is set using the CCC method (Borde and Oyama 2008; Borde et al. 2014), which keeps a direct link between the tracking step and the calculation of the AMV altitude using the individual contribution to the cross correlation of each pixel in the target box (Büche et al. 2006). The CCC method uses the cloud-top pressures (CTP) calculated for each cloudy pixel by the SAFNWC/MSG cloud-top temperature and height product (Derrien and LeGléau 2013). The final AMV altitude is the average pressure of the pixels selected by the CCC method weighted by their individual contribution to the correlation. The CTP retrieval for opaque clouds is based on the 10.8- and $12.0-\mu \mathrm{m}$ window channel brightness temperatures. For semitransparent clouds, it is based on the radiance ratioing version of the $\mathrm{CO}_{2}$ slicing technique
(Menzel et al. 1983) and the WV intercept method (Schmetz et al. 1993). The altitudes of clear-sky AMVs have been set using the CCC method together with the pixel brightness temperatures in the water vapor channel; the AMV temperature values selected by the CCC method are converted to pressure values through interpolation to the NWP model forecast temperature levels.

For both the WG and NWG configurations, the AMV altitude estimation through the mentioned height assignment techniques, commonly used to set the AMV altitude by different AMV algorithms, has been used in the same way.

\section{Results}

Table 1 shows the number of valid AMVs (with a QI larger than $85 \%$ ) produced for each MSG/SEVIRI channel for the WG and NWG configurations, and the percent difference in the number of AMVs between them [percent difference (\% Diff)].

The number of valid AMVs is generally smaller for the NWG configuration than for the WG configuration for all the MSG/SEVIRI channels when the temporal gaps are large, and especially when using small target boxes. It is instead larger for the NWG configuration in a few cases with short temporal gaps, especially for the 
TABLE 4. As in Table 1, but for the NBIAS against 1200 UTC RS wind observations. Difference in the absolute value of the NBIAS, for the NWG configuration with respect to the WG configuration for the different experiments is also shown (rightmost column).

\begin{tabular}{|c|c|c|c|c|c|c|c|c|c|}
\hline & $8 \times 8$ & $24 \times 24$ & $40 \times 40$ & $8 \times 8$ & $24 \times 24$ & $40 \times 40$ & $8 \times 8$ & $24 \times 24$ & $40 \times 40$ \\
\hline & \multicolumn{3}{|c|}{ HRVIS WG } & \multicolumn{3}{|c|}{ HRVIS NWG } & \multicolumn{3}{|c|}{ HRVIS Diff } \\
\hline 5 & -0.09 & -0.12 & -0.13 & -0.09 & -0.12 & -0.13 & -0.00 & +0.00 & -0.00 \\
\hline 15 & -0.08 & -0.10 & -0.11 & -0.06 & -0.10 & -0.11 & +0.02 & +0.00 & +0.00 \\
\hline 30 & -0.08 & -0.11 & -0.13 & +0.01 & -0.09 & -0.11 & +0.09 & +0.02 & +0.02 \\
\hline 60 & \multicolumn{3}{|c|}{ VIS08 WG } & \multicolumn{3}{|c|}{ VIS08 NWG } & +0.17 & VIS08 Diff & +0.01 \\
\hline 5 & -0.05 & -0.11 & -0.15 & -0.06 & -0.12 & -0.14 & -0.01 & -0.01 & +0.01 \\
\hline 15 & -0.14 & -0.19 & -0.22 & -0.13 & -0.18 & -0.22 & +0.01 & +0.01 & +0.00 \\
\hline 30 & -0.13 & -0.19 & -0.24 & -0.10 & -0.18 & -0.23 & +0.03 & +0.01 & +0.01 \\
\hline 60 & \multicolumn{2}{|c|}{ IR108 WG } & -0.20 & -0.01 & $\begin{array}{c}-0.18 \\
\text { IR108 NV }\end{array}$ & -0.18 & \multicolumn{2}{|c|}{ IR108 Diff } & +0.02 \\
\hline 5 & -0.01 & -0.07 & -0.11 & -0.01 & -0.07 & -0.10 & +0.00 & -0.00 & +0.01 \\
\hline 15 & -0.09 & -0.13 & -0.16 & -0.08 & -0.12 & -0.14 & +0.01 & +0.01 & +0.02 \\
\hline 30 & -0.08 & -0.12 & -0.14 & -0.05 & -0.10 & -0.13 & +0.03 & +0.02 & +0.01 \\
\hline \multirow[t]{2}{*}{60} & -0.08 & -0.12 & -0.15 & -0.01 & -0.10 & -0.13 & +0.07 & +0.02 & +0.02 \\
\hline & \multicolumn{3}{|c|}{ WV062 cloudy WG } & \multicolumn{3}{|c|}{ WV062 cloudy NWG } & \multicolumn{3}{|c|}{ WV062 cloudy Diff } \\
\hline 5 & +0.02 & -0.01 & -0.04 & +0.02 & -0.02 & -0.04 & -0.00 & -0.01 & -0.00 \\
\hline 15 & -0.04 & -0.07 & -0.09 & -0.04 & -0.05 & -0.08 & +0.00 & +0.02 & +0.01 \\
\hline 30 & -0.05 & -0.06 & -0.09 & -0.03 & -0.05 & -0.07 & +0.02 & +0.01 & +0.02 \\
\hline \multirow[t]{2}{*}{60} & -0.07 & -0.08 & -0.10 & -0.00 & -0.06 & -0.08 & +0.07 & +0.02 & +0.02 \\
\hline & \multicolumn{3}{|c|}{ WV062 clear-air WG } & \multicolumn{3}{|c|}{ WV062 clear-air NWG } & \multicolumn{3}{|c|}{ WV062 clear-air Diff } \\
\hline 5 & +0.01 & -0.03 & -0.05 & +0.01 & -0.04 & -0.06 & -0.00 & -0.01 & -0.01 \\
\hline 15 & +0.00 & -0.07 & -0.10 & +0.01 & -0.06 & -0.09 & +0.01 & +0.01 & +0.01 \\
\hline 30 & -0.01 & -0.07 & -0.11 & +0.01 & -0.07 & -0.09 & +0.02 & +0.00 & +0.02 \\
\hline 60 & -0.05 & -0.10 & -0.13 & -0.04 & -0.11 & -0.13 & +0.01 & -0.01 & -0.00 \\
\hline
\end{tabular}

VIS0.8, IR10.8, and WV6.2 channels. The increase of AMVs in the NWG configuration is only significant (over $15 \%$ difference) with a temporal gap of $5 \mathrm{~min}$, especially for the VIS0.8 and IR10.8 channels.

The differences are largest using the longest temporal gap and the smallest target box for which the reduction in the number of valid AMVs in the NWG configuration reaches a maximum of $89 \%$. The number of valid AMVs is considered to demonstrate that the other statistical parameters-mean AMV correlation, mean AMV quality index, mean AMV height, mean AMV speed, normalized bias (NBIAS), and normalized root-meansquare vector difference (NRMSVD)—have been estimated on a comparable set of data for both the WG and NWG configurations, but it is not a pertinent parameter by itself for the quality of the AMVs.

The mean tracking correlation coefficient shown in Table 2 is $0 \%-1.6 \%$ larger for the NWG configurations than for the corresponding WG configurations. The largest mean tracking correlation coefficient differences between the WG and NWG configurations occur for small target boxes and large temporal gaps. For example, the mean tracking correlation coefficient differences are $0.3 \%$ and $1.4 \%$ larger for the $\mathrm{NWG}$ configuration than for the WG configuration for the
HRVIS channel, when considering 15-min temporal gaps and using the $24 \times 24$ and $8 \times 8$ pixel target boxes, respectively. However, these two NWG configurations extract $16 \%$ and $72 \%$ less AMVs, respectively, than the corresponding WG configurations (Table 1).

It may appear difficult to explain why the WG can produce more AMVs than the NWG in some configurations, as the degree of freedom to find a good matching in the second image is much larger for the NWG configuration than for the WG configuration. The process in the NWG configuration should at least find the good solutions found in the WG configuration, plus additional cases for which the WG configuration was not able to find the correct solution due to its additional constraints: smaller search box, bad location of the search area, and error in the altitude estimation.

Figure 2 illustrates the number of WG and NWG AMV distributions as a function of the QI for a temporal gap of $15 \mathrm{~min}$ and several target boxes-40 $\times 40,24 \times 24$, and $8 \times 8$ pixels. Only AMVs having a QI $>85$ were stored when the NWCSAF HRW software was run for all the configurations; therefore, the range of the QI values on the plots is limited. The calculation of the final QI is mainly driven by spatial and temporal consistency tests (Holmlund 1998), which check that each AMV is in good agreement with its neighboring AMVs in the 
TABLE 5. As in Table 1, but for the NBIAS against the 1200 UTC NWP model wind analysis. Difference in the absolute value of the NBIAS, for the NWG configuration with respect to the WG configuration for the different experiments is also shown (rightmost column).

\begin{tabular}{|c|c|c|c|c|c|c|c|c|c|}
\hline & $8 \times 8$ & $24 \times 24$ & $40 \times 40$ & $8 \times 8$ & $24 \times 24$ & $40 \times 40$ & $8 \times 8$ & $24 \times 24$ & $40 \times 40$ \\
\hline & \multicolumn{3}{|c|}{ HRVIS WG } & \multicolumn{3}{|c|}{ HRVIS NWG } & \multicolumn{3}{|c|}{ HRVIS Diff } \\
\hline 5 & -0.09 & -0.11 & -0.12 & -0.08 & -0.11 & -0.12 & +0.01 & +0.00 & +0.00 \\
\hline 15 & -0.10 & -0.10 & -0.11 & -0.05 & -0.09 & -0.10 & +0.05 & +0.01 & +0.01 \\
\hline 30 & -0.11 & -0.12 & -0.13 & +0.03 & -0.10 & -0.11 & +0.14 & +0.02 & +0.02 \\
\hline 60 & \multicolumn{3}{|c|}{ VIS08 WG } & \multicolumn{3}{|c|}{ VIS08 NWG } & \multicolumn{3}{|c|}{ VIS08 Diff } \\
\hline 5 & -0.08 & -0.13 & -0.15 & -0.07 & -0.12 & -0.15 & +0.01 & +0.01 & +0.00 \\
\hline 15 & -0.13 & -0.17 & -0.19 & -0.11 & -0.16 & -0.18 & +0.02 & +0.01 & +0.01 \\
\hline 30 & -0.13 & -0.17 & -0.19 & -0.09 & -0.16 & -0.18 & +0.04 & +0.01 & +0.01 \\
\hline 60 & \multicolumn{2}{|c|}{ IR108 WG } & -0.20 & -0.02 & $\begin{array}{c}-0.16 \\
\text { IR108 NW }\end{array}$ & -0.18 & \multicolumn{2}{|c|}{ IR108 Diff } & +0.02 \\
\hline 5 & -0.04 & -0.09 & -0.11 & -0.03 & -0.08 & -0.10 & +0.01 & +0.01 & +0.01 \\
\hline 15 & -0.07 & -0.10 & -0.12 & -0.05 & -0.09 & -0.10 & +0.02 & +0.01 & +0.02 \\
\hline 30 & -0.08 & -0.10 & -0.12 & -0.03 & -0.08 & -0.10 & +0.05 & +0.02 & +0.02 \\
\hline \multirow[t]{2}{*}{60} & -0.12 & -0.13 & -0.14 & -0.00 & -0.08 & -0.12 & +0.12 & +0.05 & +0.02 \\
\hline & \multicolumn{3}{|c|}{ WV062 cloudy WG } & \multicolumn{3}{|c|}{ WV062 cloudy NWG } & \multicolumn{3}{|c|}{ WV062 cloudy Diff } \\
\hline 5 & +0.01 & -0.01 & -0.04 & +0.01 & -0.02 & -0.04 & -0.00 & -0.01 & -0.00 \\
\hline 15 & -0.02 & -0.04 & -0.06 & -0.01 & -0.03 & -0.05 & +0.01 & +0.01 & +0.01 \\
\hline 30 & -0.04 & -0.05 & -0.07 & -0.00 & -0.03 & -0.05 & +0.04 & +0.02 & +0.02 \\
\hline \multirow[t]{2}{*}{60} & -0.09 & -0.08 & -0.09 & +0.01 & -0.05 & -0.07 & +0.10 & +0.03 & +0.02 \\
\hline & \multicolumn{3}{|c|}{ WV062 clear-air WG } & \multicolumn{3}{|c|}{ WV062 clear-air NWG } & \multicolumn{3}{|c|}{ WV062 clear-air Diff } \\
\hline 5 & -0.00 & -0.05 & -0.06 & +0.00 & -0.06 & -0.08 & +0.00 & -0.01 & -0.02 \\
\hline 15 & -0.03 & -0.08 & -0.11 & +0.01 & -0.06 & -0.09 & +0.04 & +0.02 & +0.02 \\
\hline 30 & -0.07 & -0.09 & -0.12 & +0.00 & -0.06 & -0.09 & +0.07 & +0.03 & +0.03 \\
\hline 60 & -0.11 & -0.12 & -0.14 & -0.03 & -0.10 & -0.13 & +0.08 & +0.02 & +0.01 \\
\hline
\end{tabular}

current and previous slots. Distributions obtained for the large $40 \times 40$ pixel target boxes are very similar for the WG and NWG configurations. However, slightly more AMVs are extracted using the NWG configuration for very high QIs, those larger than 95. The tracking is normally easier using large target boxes because there is good contrast and entropy within the target box to select a good tracer and to follow it in the later images. Therefore, it is consistent to get a very similar behavior for both the WG and NWG distributions using $40 \times 40$ pixel target boxes. The extraction of slightly more NWG AMVs for very high QIs can be explained by the additional constraints of the WG configurations, which prevent the algorithm from finding the good solution because of a poor wind guess and, consequently, a bad location of the search area.

When reducing the target box size, the selection of a good tracer is more difficult, and the tracking is known to be noisier (Sohn and Borde 2008). The distributions obtained for the $24 \times 24$ pixel target boxes in Fig. 2 are also very similar for the WG and NWG configurations, but the WG configurations extract slightly more AMVs than the NWG configurations, except for a QI larger than 98 , for which a very good matching is found. Considering small $8 \times 8$ pixel target boxes, the distributions plotted in Fig. 2 show that the WG configurations extract systematically many more AMVs than the corresponding NWG configurations. All these examples illustrate that the impact of the wind guess on the tracking is really important, and when the tracking conditions are more difficult due to long temporal gaps or small target boxes, the results show that most of the valid AMVs $(\mathrm{QI}>85 \%)$ cannot be extracted without the help of the wind guess.

This behavior can be explained by the spatial and temporal consistency tests used in the quality control. The use of the wind guess in the WG configuration, which limits the search area size, does not necessary find more real good AMVs but produces more AMVs that are in quite good agreement together (having high values of the spatial and temporal quality consistency tests), and that are also in quite good agreement with the wind guess (having high values of the forecast quality consistency test). These AMVs are finally set to a good QI in the WG configuration, while the noisier NWG process does not set them to a good QI.

Table 3 shows the mean AMV speed $\left(\mathrm{m} \mathrm{s}^{-1}\right)$ for all studied configurations. Note that the mean AMV speeds are generally faster using small target boxes for both the WG and NWG configurations. This is in good agreement with the results presented in Bresky et al. (2012) and Sohn and Borde (2008). Mean AMV speeds are also 
TABLE 6. As in Table 1, but for the NRMSVD against 1200 UTC RS wind observations. Difference in the NRMSVD for the NWG configuration with respect to the WG configuration for the different experiments is also shown (rightmost column).

\begin{tabular}{|c|c|c|c|c|c|c|c|c|c|}
\hline & $8 \times 8$ & $24 \times 24$ & $40 \times 40$ & $8 \times 8$ & $24 \times 24$ & $40 \times 40$ & $8 \times 8$ & $24 \times 24$ & $40 \times 40$ \\
\hline & \multicolumn{3}{|c|}{ HRVIS WG } & \multicolumn{3}{|c|}{ HRVIS NWG } & \multicolumn{3}{|c|}{ HRVIS \%Diff } \\
\hline 5 & 0.45 & 0.41 & 0.39 & 0.44 & 0.41 & 0.39 & $-2 \%$ & $+0 \%$ & $+1 \%$ \\
\hline 15 & 0.45 & 0.38 & 0.37 & 0.42 & 0.37 & 0.36 & $-6 \%$ & $-2 \%$ & $-2 \%$ \\
\hline 30 & 0.53 & 0.38 & 0.39 & 0.50 & 0.37 & 0.37 & $-6 \%$ & $-3 \%$ & $-5 \%$ \\
\hline 60 & \multicolumn{3}{|c|}{ VIS08 WG } & \multicolumn{3}{|c|}{ VIS08 NWG } & \multicolumn{3}{|c|}{ VIS08 \%Diff } \\
\hline 5 & 0.45 & 0.44 & 0.43 & 0.45 & 0.44 & 0.44 & $+0 \%$ & $+1 \%$ & $+2 \%$ \\
\hline 15 & 0.47 & 0.47 & 0.49 & 0.46 & 0.47 & 0.48 & $-2 \%$ & $-1 \%$ & $-1 \%$ \\
\hline 30 & 0.47 & 0.48 & 0.51 & 0.46 & 0.47 & 0.51 & $-3 \%$ & $-2 \%$ & $+1 \%$ \\
\hline 60 & \multicolumn{3}{|c|}{ IR108 WG } & \multicolumn{3}{|c|}{ IR108 NWG } & $-6 \%$ & \multicolumn{2}{|c|}{ IR108 \% Diff } \\
\hline 5 & 0.43 & 0.41 & 0.41 & 0.43 & 0.41 & 0.41 & $-2 \%$ & $-1 \%$ & $-1 \%$ \\
\hline 15 & 0.42 & 0.40 & 0.40 & 0.40 & 0.39 & 0.39 & $-3 \%$ & $-2 \%$ & $-3 \%$ \\
\hline 30 & 0.42 & 0.45 & 0.40 & 0.38 & 0.38 & 0.38 & $-11 \%$ & $-14 \%$ & $-4 \%$ \\
\hline \multirow[t]{2}{*}{60} & 0.48 & 0.42 & 0.42 & 0.46 & 0.40 & 0.37 & $-3 \%$ & $-4 \%$ & $-12 \%$ \\
\hline & \multicolumn{3}{|c|}{ WV062 cloudy WG } & \multicolumn{3}{|c|}{ WV062 cloudy NWG } & \multicolumn{3}{|c|}{ WV062 cloudy \%Diff } \\
\hline 5 & 0.42 & 0.39 & 0.37 & 0.43 & 0.39 & 0.38 & $+1 \%$ & $+0 \%$ & $+3 \%$ \\
\hline 15 & 0.40 & 0.40 & 0.35 & 0.39 & 0.39 & 0.35 & $-1 \%$ & $-4 \%$ & $-1 \%$ \\
\hline 30 & 0.41 & 0.38 & 0.36 & 0.37 & 0.35 & 0.35 & $-11 \%$ & $-8 \%$ & $-2 \%$ \\
\hline \multirow[t]{2}{*}{60} & 0.47 & 0.45 & 0.39 & 0.46 & 0.38 & 0.38 & $-3 \%$ & $-14 \%$ & $-4 \%$ \\
\hline & \multicolumn{3}{|c|}{ WV062 clear-air WG } & \multicolumn{3}{|c|}{ WV062 clear-air NWG } & \multicolumn{3}{|c|}{ WV062 clear-air \% Diff } \\
\hline 5 & 0.45 & 0.42 & 0.39 & 0.44 & 0.42 & 0.39 & $-2 \%$ & $+1 \%$ & $-0 \%$ \\
\hline 15 & 0.44 & 0.41 & 0.40 & 0.45 & 0.42 & 0.40 & $+3 \%$ & $+1 \%$ & $+0 \%$ \\
\hline 30 & 0.43 & 0.41 & 0.38 & 0.47 & 0.40 & 0.38 & $+7 \%$ & $-2 \%$ & $+0 \%$ \\
\hline 60 & 0.45 & 0.42 & 0.40 & 0.44 & 0.41 & 0.40 & $-1 \%$ & $-0 \%$ & $+0 \%$ \\
\hline
\end{tabular}

generally faster for small temporal gaps, except when using small target boxes. Considering 30- or 60-min temporal gaps, only the most persistent and well-identified features are successfully tracked. This means that the cloud shapes do not change too much in the consecutive images, and that the target boxes contain enough contrast to identify a good tracer. These conditions are very difficult to meet using very small target boxes, which is the reason why the numbers of AMVs in Table 1 drop to a few cases for such configurations and give extreme mean AMV speed statistics-for example, $44.6 \mathrm{~m} \mathrm{~s}^{-1}$ for the HRVIS channel; $8 \times 8$ pixel target boxes and 60 -min temporal gaps, $27.8 \mathrm{~m} \mathrm{~s}^{-1}$ for the VIS08 channel; and $33.8 \mathrm{~m} \mathrm{~s}^{-1}$ for the IR108 channel considering the NWG configuration. These AMVs are generally found at higher altitudes, which explains the high mean speeds. For example, the NWG HRVIS AMVs extracted using $8 \times 8$ pixel target boxes and 60-min temporal gaps are found on average $\sim 100$ and $\sim 180 \mathrm{hPa}$ higher than the ones extracted using 30- and 5-min temporal gaps, respectively.

The general behavior as a function of target box sizes or temporal gaps is similar for the WG and NWG configurations, but the mean AMV speed is higher in the NWG configurations than in the WG configurations for most cases. Accordingly, the AMVs extracted in the
NWG configurations are found a few hectopascals higher in the troposphere than the AMVs extracted in the WG configurations. The analysis of the corresponding SAFNWC/MSG cloud-type products shows that the NWG configurations tend to extract a few more AMVs at high levels than the corresponding WG configurations, and it is the opposite at low levels. Unfortunately, the SAFNWC/MSG cloud-type product does not differentiate between stratus and cumulus clouds, preventing a detailed study on the specific role of the convection (Bedka and Mecikalski 2005) on AMV extraction in the NWG and WG configurations. However, the statistics presented here does not clearly indicate that convection has a significant impact that can explain the differences between the WG and NWG results. The results simply show that NWG AMVs are, on average, based on tracers that appear more stable over time.

Corresponding to the higher mean AMV speed, the NBIAS is smaller in most cases for the NWG configuration. The impact is a bit smaller when compared to the radiosonde winds (Table 4) than when compared to the NWP model wind analysis (Table 5), but the same general trends are observed. The reduction of the NBIAS is larger for the longest temporal gaps and the smallest target boxes, but it corresponds to small sets of 
TABLE 7. As in Table 1, but for the NRMSVD against 1200 UTC NWP model wind analysis. Percent difference in the NRMSVD for the NWG configuration with respect to the WG configuration for the different experiments is also shown (rightmost column).

\begin{tabular}{|c|c|c|c|c|c|c|c|c|c|}
\hline & $8 \times 8$ & $24 \times 24$ & $40 \times 40$ & $8 \times 8$ & $24 \times 24$ & $40 \times 40$ & $8 \times 8$ & $24 \times 24$ & $40 \times 40$ \\
\hline & \multicolumn{3}{|c|}{ HRVIS WG } & \multicolumn{3}{|c|}{ HRVIS NWG } & \multicolumn{3}{|c|}{ HRVIS \%Diff } \\
\hline 5 & 0.34 & 0.30 & 0.28 & 0.31 & 0.30 & 0.28 & $-8 \%$ & $-1 \%$ & $-1 \%$ \\
\hline 15 & 0.39 & 0.27 & 0.27 & 0.34 & 0.25 & 0.25 & $-13 \%$ & $-7 \%$ & $-6 \%$ \\
\hline 30 & 0.49 & 0.30 & 0.30 & 0.45 & 0.26 & 0.26 & $-10 \%$ & $-13 \%$ & $-11 \%$ \\
\hline 60 & \multicolumn{3}{|c|}{ VIS08 WG } & \multicolumn{3}{|c|}{ VIS08 NWG } & \multicolumn{3}{|c|}{ VIS08 \%Diff } \\
\hline 5 & 0.37 & 0.35 & 0.35 & 0.36 & 0.35 & 0.34 & $-2 \%$ & $-0 \%$ & $-0 \%$ \\
\hline 15 & 0.36 & 0.36 & 0.36 & 0.33 & 0.35 & 0.35 & $-9 \%$ & $-4 \%$ & $-4 \%$ \\
\hline 30 & 0.38 & 0.36 & 0.37 & 0.35 & 0.34 & 0.36 & $-8 \%$ & $-7 \%$ & $-5 \%$ \\
\hline 60 & \multicolumn{3}{|c|}{ IR108 WG } & \multicolumn{3}{|c|}{ IR108 NWG } & \multicolumn{3}{|c|}{ IR108 \%Diff } \\
\hline 5 & 0.34 & 0.32 & 0.31 & 0.32 & 0.30 & 0.29 & $-5 \%$ & $-5 \%$ & $-6 \%$ \\
\hline 15 & 0.29 & 0.27 & 0.27 & 0.25 & 0.24 & 0.24 & $-12 \%$ & $-9 \%$ & $-11 \%$ \\
\hline 30 & 0.31 & 0.26 & 0.27 & 0.27 & 0.23 & 0.24 & $-13 \%$ & $-13 \%$ & $-12 \%$ \\
\hline \multirow[t]{2}{*}{60} & 0.43 & 0.33 & 0.33 & 0.39 & 0.29 & 0.29 & $-8 \%$ & $-12 \%$ & $-11 \%$ \\
\hline & \multicolumn{3}{|c|}{ WV062 cloudy WG } & \multicolumn{3}{|c|}{ WV062 cloudy NWG } & \multicolumn{3}{|c|}{ WV062 cloudy \% Diff } \\
\hline 5 & 0.29 & 0.27 & 0.25 & 0.29 & 0.27 & 0.26 & $-1 \%$ & $+1 \%$ & $+2 \%$ \\
\hline 15 & 0.23 & 0.22 & 0.21 & 0.22 & 0.21 & 0.20 & $-7 \%$ & $-5 \%$ & $-6 \%$ \\
\hline 30 & 0.28 & 0.23 & 0.24 & 0.24 & 0.21 & 0.21 & $-12 \%$ & $-12 \%$ & $-12 \%$ \\
\hline \multirow[t]{2}{*}{60} & 0.40 & 0.30 & 0.31 & 0.37 & 0.26 & 0.27 & $-8 \%$ & $-13 \%$ & $-14 \%$ \\
\hline & \multicolumn{3}{|c|}{ WV062 clear-air WG } & \multicolumn{3}{|c|}{ WV062 clear-air NWG } & \multicolumn{3}{|c|}{ WV062 clear-air \% Diff } \\
\hline 5 & 0.31 & 0.30 & 0.29 & 0.30 & 0.30 & 0.30 & $-5 \%$ & $+2 \%$ & $+3 \%$ \\
\hline 15 & 0.28 & 0.28 & 0.28 & 0.26 & 0.27 & 0.27 & $-7 \%$ & $-4 \%$ & $-4 \%$ \\
\hline 30 & 0.33 & 0.29 & 0.29 & 0.30 & 0.27 & 0.27 & $-9 \%$ & $-7 \%$ & $-7 \%$ \\
\hline 60 & 0.38 & 0.32 & 0.32 & 0.35 & 0.30 & 0.31 & $-6 \%$ & $-5 \%$ & $-4 \%$ \\
\hline
\end{tabular}

very specific tracers found at very high levels, as explained above.

The NRMSVD against radiosonde winds and the NWP model wind analysis are shown in Tables 6 and 7, respectively. The trend observed for the NBIAS is also observed in the NRMSVD, and values for both are smaller for NWG configurations. This effect is slightly larger when AMVs are compared against the NWP model wind analysis. The largest reductions of NRMSVD are around $15 \%$ and occur using an intermediate temporal gap of $30 \mathrm{~min}$ for all channels.

\section{Discussion}

The results obtained in the study show that the statistics of AMVs extracted from NWC SAF/HRW software against radiosonde wind observations and NWP model wind analyses are better when the wind guess is not used to locate the search area. Both NBIAS and NRMSVD are generally smaller when the wind guess has not been used even for difficult tracking conditions like small target boxes or long temporal gaps.

The total number of valid AMVs is larger for the WG configuration than for the NWG configuration, especially when small target boxes or long temporal gaps are used. AMVs are more numerous for the WG configuration because the tracking is less noisy for such difficult tracking conditions, but they have worse performances than NWG AMVs when they are compared to both radiosonde wind observations and NWP model wind analyses. For large target boxes and short temporal gaps, NWG configurations extract a few more AMVs than WG configurations. In regions where the wind shear is important, an error in the estimation of the tracer altitude is transferred to the interpolation of the wind guess speed and direction from the NWP forecast fields. Both types of errors can prevent the algorithm from finding the tracer in the later image because the search area defined by the NWP wind guess is not located at the correct position. Therefore, the correct matching solution is indeed outside the search area, and it cannot be found by the WG configurations. According to the way the quality consistency tests are used in the calculation of the final QI, all these results show that some of the AMVs extracted in the WG configurations cannot be extracted without the help of the wind guess, and that these AMVs hence contain significant information from the wind guess. This is an important result that must be considered carefully by AMV producers, especially in the framework of deriving smaller-scale 
information to feed regional forecast models using smaller target boxes.

Based on these results, we judge that it is better to avoid the reference to the model fields in the AMV extraction schemes. This comment concerns the use of the wind guess to help the tracking and also the reference to the forecast consistency in the calculation of the final QI. A more powerful computing environment can always be considered to ensure the operational extraction of AMVs without the help of the wind guess.

The results presented in this paper also illustrate the role of the actual quality control scheme used in AMV extraction algorithms, which is mainly based on spatial and temporal consistency checks. These tests ensure that a valid AMV is in good agreement with neighboring AMVs in the current and previous slot. Such definition of a valid AMV forces the valid AMVs to be part of large flows to be set to a good QI. This definition of goodquality AMV is certainly appropriate for global-scale NWP models, but it becomes a strong limitation when extracting the smaller-scale information that is expected by the AMV producers using very small target boxes. Indeed, the risk of applying the current quality control approach associated with the use of wind guess is that only information present in the model is reproduced, and nothing else. These aspects will undoubtedly be discussed in the context of different quality control approaches for AMVs-weighting down the role of spatial consistency, for example - that enable the extraction of smaller-scale information appropriate to regional NWP models.

\section{REFERENCES}

Bedka, K., and J. R. Mecikalski, 2005: Application of satellitederived atmospheric motion vectors for estimating mesoscale flows. J. Appl. Meteor., 44, 1761-1772.

Borde, R., and R. Oyama, 2008: A direct link between feature tracking and height assignment of operational atmospheric motion vectors. Proc. Ninth Int. Winds Workshop, Annapolis, MD, EUMETSAT, Paper 51_S3_13. [Available online at https:// www.eumetsat.int/website/home/News/DAT_2042907.html.] , and P. Dubuisson, 2010: Sensitivity of atmospheric motion vectors height assignment methods to semitransparent cloud properties using simulated Meteosat- 8 radiances. J. Appl. Meteor. Climatol., 49, 1205-1218.

_ M. Doutriaux-Boucher, G. Dew, and M. Carranza, 2014: A direct link between feature tracking and height assignment of operational EUMETSAT atmospheric motion vectors. $J$. Atmos. Oceanic Technol., in press.

Bresky, W. C., J. M. Daniels, A. A. Bailey, and S. T. Wanzong, 2012: New methods toward minimizing the slow speed bias associated with atmospheric motion vectors. J. Appl. Meteor. Climatol., 51, 2137-2151.

Büche, G., H. Karbstein, A. Kummer, and H. Fischer, 2006: Water vapor structure displacements from cloud-free Meteosat scenes and their interpretation for the wind field. J. Appl. Meteor., 45, 556-575.

Derrien, M., and H. LeGléau, 2013: Algorithm theoretical basis document for "cloud products" (CMa-PGE01 v3.2, CT-PGE02 v2.2 and CTTH-PGE03 v2.2). NWC SAF Doc. SAF/NWC/ CDOP2/MFL/SCI/ATBD/01, Issue 3.2.1, 87 pp. [Available online at http://www.nwcsaf.org/indexScientificDocumentation. html.]

García-Pereda, J., 2013a: Algorithm theoretical basis document for "high resolution winds" (HRW - PGE09 v4.0). NWC SAF Doc. SAF/NWC/CDOP/INM/SCI/ATBD/09, Issue 4.0, 78 pp. [Available online at http://www.nwcsaf.org/indexScientific Documentation.html.] 2013b: Validation report for "high resolution winds" (HRW PGE09 v4.0). NWC SAF Doc. SAF/NWC/CDOP/INM/SCI/ VR/13, Issue 1.0, $30 \mathrm{pp}$. [Available online at http://www.nwcsaf. org/indexScientificDocumentation.html.]

Holmlund, K., 1998: The utilization of statistical properties of satellite-derived atmospheric motion vectors to derive quality indicators. Wea. Forecasting, 13, 281-288.

Menzel, W. P., W. L. Smith, and T. R. Stewart, 1983: Improved cloud motion wind vector and altitude assignment using VAS. J. Climate Appl. Meteor., 22, 377-384.

Schmetz, J., K. Holmlund, J. Hoffman, B. Strauss, B. Mason, V. Gaertner, A. Koch, and L. van de Berg, 1993: Operational cloud motion winds from Meteosat infrared images. J. Appl. Meteor., 32, 1206-1225.

Sohn, E. H., and R. Borde, 2008: The impact of window size on AMVs. Proc. Ninth Int. Winds Workshop, Annapolis, MD, EUMETSAT, Paper 51_S4_18. [Available online at https://www.eumetsat.int/website/home/News/DAT_2042907. html.]

Velden, C., S. J. Nieman, W. P. Menzel, S. T. Wanzong, and J. S. Goerss, 1997: Upper tropospheric winds derived from geostationary satellite water vapor observations. Bull. Amer. Meteor. Soc., 78, 173-195. 